\title{
Mass splittings in a linear sigma model for multiflavor gauge theories
}

\author{
D. Floor, E. Gustafson, ${ }^{*}$ and Y. Meurice \\ Department of Physics and Astronomy, The University of Iowa, Iowa City, Iowa 52242, USA
}

(Received 10 August 2018; published 19 November 2018)

\begin{abstract}
We calculate the tree-level mass spectrum for a linear sigma model describing the scalar and pseudoscalar mesons of a $S U(3)$ local gauge theory with Dirac fermions in the fundamental representation. $N_{1}$ fermions have a mass $m_{1}$ and $N_{2}$ a mass $m_{2}$. Using recent lattice data with $m_{1}=m_{2}$ and $N_{1}+N_{2}=8$ or 12 , we predict the mass splittings for $m_{2}=m_{1}+\delta m$. At first order in $\delta m$, an interesting inverted pattern appears in the $0^{++}$sector, where mesons with lighter fermions are heavier. This feature could be tested in ongoing calculations provided that $m_{1}$ and $\delta m$ are sufficiently small. We discuss possible improvements of the approach.
\end{abstract}

DOI: 10.1103/PhysRevD.98.094509

\section{INTRODUCTION}

The idea that asymptotically free gauge theories with a sufficiently large number of massless fermions (flavors) have a nontrivial infrared fixed point (IRFP) has motivated many lattice studies [1-3]. It is expected that in the massless limit, conformal symmetry and gapless deconfined excitations are present. This limiting situation is unlikely to be directly relevant for particle physics. Nevertheless by introducing mass terms or reducing the number of flavors slightly below a critical value, one can obtain possibly interesting models in the context of electroweak symmetry breaking. If an IRFP exists, fermion masses provide relevant directions out of this IRFP which are expected to drive the renormalization group (RG) flows towards fixed points where a more conventional behavior is expected. However, a consensus on a physical picture supported by an effective theory is still lacking [1-3].

In this article we focus on the well-studied example of a $S U$ (3) gauge theory with $N_{f}$ fundamental Dirac fermions. In the massless limit, we have a clear physical picture when the $N_{f}$ is not too large, say for $N_{f} \leq 4$, the clearly QCD-like region: there are $N_{f}^{2}-1$ massless pions and the other states (scalars with positive parity, baryons, ...) are massive. At the other end, for $N_{f}=16$, the last value preserving asymptotic freedom, the two-loop beta function has a nontrivial zero at $\alpha_{c} \simeq 1 / 20$ and perturbation theory should be valid to describe weakly interacting massless

\footnotetext{
* Corresponding author. erik-j-gustafson@uiowa.edu

Published by the American Physical Society under the terms of the Creative Commons Attribution 4.0 International license. Further distribution of this work must maintain attribution to the author(s) and the published article's title, journal citation, and DOI. Funded by SCOAP ${ }^{3}$.
}

deconfined quarks and gluons. It is clear that as $N_{f}$ is increased between these two limits, the low energy degrees of freedom change drastically, however a consensus on the details of the changes is not available so far. One important limitation is that lattice simulations with low fermion masses are typically impractical and one has to rely on models for the massless extrapolation. Nevertheless there is a consensus on the fact that adding light flavors tend to produce unexpectedly light states besides the pions.

Light $\sigma$ masses were found for $S U(3)$ gauge theories with 8 [4-7] and 12 [6,8] fundamental flavors and also for 2 sextets [9]. Recent results [10,11] concerning the mass of the $\eta^{\prime}$ support the possibility [12] that the explicit breaking of the axial $U(1)_{A}$ symmetry, which depends in a distinct way on $N_{f}$, can explain the fact that the $\sigma$ becomes lighter as $N_{f}$ increases. One simple and interesting possibility [13-15] is that at some point $N_{f}$ reaches the boundary of the conformal window which is signaled by the fact that the $\sigma$ and other states become massless. However, more complex intermediate situations or phases are conceivable when $N_{f}$ is increased.

An interesting question is to figure out if unexpectedly light states persist when a certain number of fermions have a larger mass. In the following, we consider a linear sigma model with $N_{1}$ light hyperquarks of mass $m_{1}$ and $N_{2}$ heavier hyperquarks of mass $m_{2}$. This model is an extension of the single mass model discussed in Ref. [12] and which was introduced and studied in QCD context with several variations [16-26]. The case $N_{1}=2$, $N_{2} \geq 4$ could provide interesting extensions of the minimal technicolor scenario [27] but it has not been studied on the lattice so far because multiples of 4 are convenient with staggered fermions. Part of the spectrum for $N_{1}=4$, $N_{2}=8$ has been extracted from recent lattice simulations 
[28,29]. A possible phenomenological motivation for this choice is given in Ref. [30].

The article is organized as follows. The linear sigma model is presented in Sec. II. The tree-level spectrum is calculated in Sec. III. In the one fermion mass case, $2 N_{f}^{2}$ bosons are characterized by 4 masses $\left(\sigma, \mathbf{a}_{0}, \eta^{\prime}\right.$ and $\left.\boldsymbol{\pi}\right)$. With two fermion masses, the $\mathbf{a}_{\mathbf{0}}$ and $\boldsymbol{\pi}$ each split into four representations of the unbroken subgroup $S U\left(N_{1}\right)_{V} \otimes$ $S U\left(N_{2}\right)_{V}$. Section IV discusses the determination of the parameters of the model in terms of the masses in the equal mass case. We emphasize that if we want to fit the spectrum using the tree level model, the quartic couplings depend on the symmetry breaking term in a way that is only understood empirically. In Sec. V we introduce a perturbative approach where $m_{2}=m_{1}+\delta m$ and calculate the mass splitting at first order in $\delta m$. This allows us to use the empirical unperturbed (single mass) results $[6,11]$ to estimate the mass splittings. We obtain simple ratios of differences between masses squared which are identical for scalars and pseudoscalars. However, the numerical results of the LatKMI collaboration $[6,11]$ indicate that at first order there is an interesting inversion for the adjoint $0^{++}$ (the $\mathbf{a}_{\mathbf{0}}$ ), namely the meson containing light hyperquarks are heavier than those containing one or two heavy hyperquarks. In the conclusions, we discuss how to test these predictions in ongoing lattice simulations and how additional effect could modify the results.

\section{THE TWO MASS MODEL}

The model considered here is introduced and motivated in Refs. [12,16-19]. The only difference with Ref. [12] is the explicit breaking of the chiral symmetry which here corresponds to $N_{1}$ hyperquarks of mass $m_{1}$ and $N_{2}$ hyperquarks of mass $m_{2}$, with $N_{1}+N_{2}=N_{f}$. For the sake of self-containedness, some basic points are repeated below.

The effective fields $\phi_{i j}$ are $N_{f} \times N_{f}$ matrices transforming as $\bar{\psi}_{R j} \psi_{L i}$ under $U\left(N_{f}\right)_{L} \otimes U\left(N_{f}\right)_{R}$. We use the parametrization:

$$
\phi=\left(S_{\alpha}+i P_{\alpha}\right) \Gamma^{\alpha},
$$

with a summation over $\alpha=0,1, \ldots N_{f}^{2}-1$ for a basis of $N_{f} \times N_{f}$ Hermitian matrices $\Gamma^{\alpha}$ such that

$$
\operatorname{Tr}\left(\Gamma^{\alpha} \Gamma^{\beta}\right)=(1 / 2) \delta^{\alpha \beta} .
$$

We use the convention that $\Gamma_{0}=\mathbb{1}_{N_{f} \times N_{f}} / \sqrt{2 N_{f}}$ while the remaining $N_{f}^{2}-1$ matrices are traceless. The $S_{0}$ and $P_{0}$ correspond to the $\sigma$ and $\eta^{\prime}$ respectively while the remaining components transform like the adjoint representation and are denoted $\mathbf{a}_{\mathbf{0}}$ and $\boldsymbol{\pi}$ respectively. In addition, we define a $N_{f} \times N_{f}$ matrix for which we use the short notation " $\Gamma$ " " in analogy with the $2+1$ flavors case and which is defined as
$\Gamma^{8} \equiv \frac{1}{\sqrt{2 N_{f}}}\left(\begin{array}{cc}\sqrt{N_{2} / N_{1}} \mathbb{1}_{N_{1} \times N_{1}} & 0 \\ 0 & -\sqrt{N_{1} / N_{2}} \mathbb{1}_{N_{2} \times N_{2}}\end{array}\right)$

The effective Lagrangian has a canonical kinetic term

$$
\mathcal{L}_{\text {kin }}=\operatorname{Tr} \partial_{\mu} \phi \partial^{\mu} \phi^{\dagger}
$$

and a potential term consisting of three parts

$$
V=V_{0}+V_{a}+V_{m} .
$$

The first two terms are given as

$$
\begin{aligned}
V_{0} \equiv & -\mu^{2} \operatorname{Tr}\left(\phi^{\dagger} \phi\right)+(1 / 2)\left(\lambda_{\sigma}-\lambda_{a 0}\right)\left(\operatorname{Tr}\left(\phi^{\dagger} \phi\right)\right)^{2} \\
& +\left(N_{f} / 2\right) \lambda_{a 0} \operatorname{Tr}\left(\left(\phi^{\dagger} \phi\right)^{2}\right),
\end{aligned}
$$

and

$$
V_{a} \equiv-2\left(2 N_{f}\right)^{N_{f} / 2-2} X\left(\operatorname{det} \phi+\operatorname{det} \phi^{\dagger}\right) .
$$

The third term represent the effect of mass term with $N_{1}$ flavors of mass $m_{1}$ and $N_{2}$ flavors of mass $m_{2}$,

$$
V_{m} \equiv-(\operatorname{Tr} \mathcal{M} \phi+\text { H.c. })=-b_{0} S_{0}-b_{8} S_{8} .
$$

The matrix $\mathcal{M}$ can be written as $b_{0} \Gamma^{0}+b_{8} \Gamma^{8}$ and $V_{m}$ is invariant under $S U\left(N_{1}\right)_{V} \otimes S U\left(N_{2}\right)_{V}$. We assume that this vector symmetry is not broken spontaneously and that the vacuum expectation of $\phi$ has the form:

$$
\langle\phi\rangle=\frac{1}{\sqrt{2 N_{f}}} \cdot\left(\begin{array}{cc}
v_{1} \mathbb{1}_{N_{1} \times N_{1}} & 0 \\
0 & v_{2} \mathbb{1}_{N_{2} \times N_{2}}
\end{array}\right) .
$$

This means that $\left\langle S_{0}\right\rangle=v_{0}$ and $\left\langle S_{8}\right\rangle=v_{8}$ or equivalently

$$
\langle\phi\rangle=v_{0} \Gamma^{0}+v_{8} \Gamma^{8} .
$$

The transformation between the two expressions is

$$
\begin{aligned}
& v_{1}=v_{0}+\sqrt{N_{2} / N_{1}} v_{8} \\
& v_{2}=v_{0}-\sqrt{N_{1} / N_{2}} v_{8},
\end{aligned}
$$

and its inverse

$$
\begin{gathered}
v_{0}=\left(1 / N_{f}\right)\left(N_{1} v_{1}+N_{2} v_{2}\right) \\
v_{8}=\left(\sqrt{N_{1} N_{2}} / N_{f}\right)\left(v_{1}-v_{2}\right) .
\end{gathered}
$$

The same transformation can be used to define $b_{1}$ and $b_{2}$ in terms of $b_{0}$ and $b_{8}$. This implies that

$$
b_{0} S_{0}+b_{8} S_{8}=\left(N_{1} / N_{f}\right) v_{1} b_{1}+\left(N_{2} / N_{f}\right) v_{2} b_{2} \text {. }
$$


The vacuum values $v_{1}$ and $v_{2}$ are given in terms of the couplings using the minimization conditions that arise from the requirement that:

$$
\left.\frac{\partial V}{\partial S_{i}}\right|_{\langle\phi\rangle}=0, \quad i=0,8
$$

which simplify to:

$$
\begin{gathered}
M_{\pi_{l l}}^{2} v_{1}=b_{1} \\
M_{\pi_{h h}}^{2} v_{2}=b_{2},
\end{gathered}
$$

with $M_{\pi_{l l}}$ and $M_{\pi_{h h}}$, the pseudo-Nambu-Goldstone boson masses corresponding to light-light and heavy-heavy hyperquarks and discussed in the next section. The relations (17) and (18) among Nambu-Goldstone bosons masses, vacuum expectations and symmetry breaking terms follow from general identities described in (19.3.3) in [31].

The full Lagrangian involves six constants $\left(\mu^{2}, \lambda_{\sigma}, \lambda_{a 0}\right.$, $\left.\mathrm{X}, b_{0}, b_{8}\right)$. The minimization of the potential determines $v_{0}$ and $v_{8}$ (or equivalently $v_{1}$ and $v_{2}$ ) and these two vacuum expectations depend implicitly on the six constants. Before we calculate the mass spectrum, it is important to understand how numerical data can be used to determine the unknown parameters.

In the unperturbed case $\left(b_{8}=0\right)$ the spectrum has 4 independent masses $\left(M_{\pi}^{2}, M_{\eta^{\prime}}^{2}, M_{a 0}^{2}, M_{\sigma}^{2}\right)$ and 1 minimization condition, while the Lagrangian has 5 parameters $\left(\mu^{2}, \lambda_{\sigma}, \lambda_{a 0}, X, b_{0}\right)$ and 1 vacuum expectation value. The minimization condition essentially fixes the value of $b_{0}$, eliminating one of the parameters of the Lagrangian. The vacuum expectation value ( $v$ in the unperturbed single mass case) is related to the $f_{\pi}$; however it is not necessary to know what the v.e.v.'s value is because the parameters $\lambda_{\sigma}$, $\lambda_{a 0}$ and $X$ carry around factors of the v.e.v. $\left(\lambda_{\sigma} v^{2}, \lambda_{a 0} v^{2}\right.$, and $X v^{N-2}$ ) and it is possible to write down simple linear combinations of the masses to describe this phenomena. However because the number of free parameters is equal to the number of particles the model is not predictive. However, some combinations of the quantities vary slowly and interesting features appear [12]. In the case of two different fermion masses that we investigate in this paper, we add a sixth constant $b_{8}$; this introduces one more minimization condition. The Lagrangian still has 4 parameters to fit, however, the Lagrangian describes 10 different mesons. This implies that the splitting of the quark masses (or the splitting of the pions) fixes all the other meson mass splittings and we can make predictions about what will happen to the meson masses.

\section{THE SPECTRUM}

The spectrum of the model can be obtained from the second derivatives of the potential at $\langle\phi\rangle$ :

$$
\begin{aligned}
& M_{S \alpha \beta}^{2} \equiv \partial^{2} V /\left.\partial S_{\alpha} \partial S_{\beta}\right|_{\langle\phi\rangle} \\
& M_{P \alpha \beta}^{2} \equiv \partial^{2} V /\left.\partial P_{\alpha} \partial P_{\beta}\right|_{\langle\phi\rangle} .
\end{aligned}
$$

When the two masses are equal each parity sector splits into a singlet and the adjoint of $S U\left(N_{f}\right)_{V}$. We now consider the effect of having two masses with the convention $m_{1} \leq m_{2}$. We call the $N_{1}$ flavors of mass $m_{1}$ "light" and the $N_{2}$ flavors of mass $m_{2}$ "heavy". The adjoint of $S U\left(N_{f}\right)_{V}$ can be decomposed into a representation of the $S U\left(N_{1}\right)_{V} \otimes$ $S U\left(N_{2}\right)_{V}$ subgroup as follows:

$$
\left(N_{1}^{2}-1,1\right) \bigoplus\left(1, N_{2}^{2}-1\right) \bigoplus\left(\left(N_{1}, \bar{N}_{2}\right)+\text { H.c. }\right) \bigoplus(1,1) .
$$

We call the first three representations light-light $(l l)$, heavyheavy $(h h)$, heavy-light $(h l)$. The last one is the singlet associated with $\Gamma^{8}$. Except for one mixing between the indices 0 and $8, M_{S \alpha \beta}^{2}$ and $M_{P \alpha \beta}^{2}$ are diagonal.

In order to clarify the meaning of the indices $l l, h l$ and $h h$, we can consider the almost physical example of 3 flavor QCD in the isospin limit $\left(m_{u}=m_{d} \ll m_{s}\right)$. This corresponds to the case $N_{1}=2$ and $N_{2}=1$. The $l l$ form the pion triplet, the $h l$ are the two Kaon doublets and the $\eta$ is the singlet denoted $(1,1)$ above. Because $N_{2}^{2}-1=0$, there are no $h h$ in this example. In order to get $h h$ states, we could consider the fictitious case of one more flavor with $m_{s}=m_{c}$. The $h h$ states would be the $D_{s}$, the $\overline{D_{s}}$ and a linear combination of the $\eta, \eta^{\prime}$ and $\eta_{c}$.

All the diagonal terms have a common term:

$$
\mathcal{C} \equiv-\mu^{2}+\frac{\lambda_{\sigma}-\lambda_{a 0}}{2 N_{f}}\left(N_{1} v_{1}^{2}+N_{2} v_{2}^{2}\right)
$$

We now proceed to give explicit expressions for the second derivatives. For the nonsinglet pseudoscalars we have

$$
\begin{gathered}
M_{\pi_{l l}}^{2}=\mathcal{C}+\frac{\lambda_{a 0}}{2} v_{1}^{2}-\frac{X}{N_{f}} v_{1}^{N_{1}-2} v_{2}^{N_{2}} \\
M_{\pi_{l h}}^{2}-M_{\pi_{l l}}^{2}=\left(\frac{\lambda_{a 0}}{2}+\frac{X}{N_{f}} v_{1}^{N_{1}-2} v_{2}^{N_{2}-2}\right)\left(v_{2}-v_{1}\right) v_{2} \\
M_{\pi_{h h}}^{2}-M_{\pi_{l l}}^{2}=\left(\frac{\lambda_{a 0}}{2}+\frac{X}{N_{f}} v_{1}^{N_{1}-2} v_{2}^{N_{2}-2}\right)\left(v_{2}^{2}-v_{1}^{2}\right) .
\end{gathered}
$$

As explained above, the two singlets have a mixing term and the spectrum in this sector is given by the eigenvalues of a $2 \times 2$ matrix. The situation is similar to having the physical $\eta$ and $\eta^{\prime}$ given as mixings of the mathematical $S U(3)$ and $S U(2) \otimes U(1)$ singlets in three flavor QCD. 


$$
\begin{aligned}
& M_{P_{00}}^{2}=\mathcal{C}+\frac{\lambda_{a 0}}{2 N_{f}}\left(N_{1} v_{1}^{2}+N_{2} v_{2}^{2}\right)+\frac{X}{N_{f}^{2}} v_{1}^{N_{1}-2} v_{2}^{N_{2}-2}\left(\left(N_{1} v_{2}+N_{2} v_{1}\right)^{2}-\left(N_{1} v_{2}^{2}+N_{2} v_{1}^{2}\right)\right) \\
& M_{P_{88}}^{2}=\mathcal{C}+\frac{\lambda_{a 0}}{2 N_{f}}\left(N_{2} v_{1}^{2}+N_{1} v_{2}^{2}\right)+\frac{X}{N_{f}^{2}} v_{1}^{N_{1}-2} v_{2}^{N_{2}-2}\left(N_{1} N_{2}\left(v_{2}-v_{1}\right)^{2}-\left(N_{2} v_{2}^{2}+N_{1} v_{1}^{2}\right)\right) \\
& M_{P_{08}}^{2}=\left(v_{2}-v_{1}\right)\left[-\frac{\lambda_{a 0}}{2 N_{f}} \sqrt{N_{1} N_{2}}\left(v_{1}+v_{2}\right)+\frac{X}{N_{f}^{2}} v_{1}^{N_{1}-2} v_{2}^{N_{2}-2} \sqrt{N_{1} N_{2}}\left(\left(N_{1} v_{2}+N_{2} v_{1}\right)-\left(v_{2}+v_{1}\right)\right)\right] .
\end{aligned}
$$

For the non-singlet scalars, we have

$$
\begin{aligned}
& M_{a 0_{l l}}^{2}-M_{\pi_{l l}}^{2}=\lambda_{a 0} v_{1}^{2}+\frac{2 X}{N_{f}} v_{1}^{N_{1}-2} v_{2}^{N_{2}} \quad M_{a 0_{l h}}^{2}-M_{\pi_{l h}}^{2}=\lambda_{a 0} v_{1} v_{2}+\frac{2 X}{N_{f}} v_{1}^{N_{1}-1} v_{2}^{N_{2}-1} \\
& M_{a 0_{h h}}^{2}-M_{\pi_{h h}}^{2}=\lambda_{a 0} v_{2}^{2}+\frac{2 X}{N_{f}} v_{1}^{N_{1}} v_{2}^{N_{2}-2} .
\end{aligned}
$$

For the two singlets and their mixing

$$
\begin{aligned}
M_{S_{00}}^{2}= & \mathcal{C}+\frac{\lambda_{\sigma}-\lambda_{a 0}}{2 N_{f}}\left(\frac{2}{N_{f}}\left(N_{1} v_{1}+N_{2} v_{2}\right)^{2}\right)+\frac{3 \lambda_{a 0}}{2 N_{f}}\left(N_{1} v_{1}^{2}+N_{2} v_{2}^{2}\right)-\frac{X}{N_{f}^{2}} v_{1}^{N_{1}-2} v_{2}^{N_{2}-2}\left(\left(N_{1} v_{2}+N_{2} v_{1}\right)^{2}-\left(N_{1} v_{2}^{2}+N_{2} v_{1}^{2}\right)\right) \\
M_{S_{88}}^{2}= & \mathcal{C}+\frac{\lambda_{\sigma}-\lambda_{a 0}}{2 N_{f}}\left(\frac{2 N_{1} N_{2}}{N_{f}}\left(v_{1}-v_{2}\right)^{2}\right)+\frac{3 \lambda_{a 0}}{2 N_{f}}\left(N_{2} v_{1}^{2}+N_{1} v_{2}^{2}\right)-\frac{X}{N_{f}^{2}} v_{1}^{N_{1}-2} v_{2}^{N_{2}-2}\left(N_{1} N_{2}\left(v_{2}-v_{1}\right)^{2}-\left(N_{2} v_{2}^{2}+N_{1} v_{1}^{2}\right)\right) \\
M_{S_{08}}^{2}= & \left(v_{2}-v_{1}\right)\left[-\frac{\lambda_{\sigma}-\lambda_{a 0}}{N_{f}^{2}}\left(\sqrt{N_{1} N_{2}}\left(N_{1} v_{1}+N_{2} v_{2}\right)\right)-\frac{3 \lambda_{a 0}}{2 N_{f}} \sqrt{N_{1} N_{2}}\left(v_{1}+v_{2}\right)\right. \\
& \left.-\frac{X}{N_{f}^{2}} \sqrt{N_{1} N_{2}} v_{1}^{N_{1}-2} v_{2}^{N_{2}-2}\left(\left(N_{2} v_{1}+N_{1} v_{2}\right)-\left(v_{2}+v_{1}\right)\right)\right] .
\end{aligned}
$$

\section{REMARKS ABOUT THE UNPERTURBED SPECTRUM}

Before applying the equations given in Sec. III to practical situations, we need to clarify some aspects of our understanding of the unperturbed model, in other words, with $m_{f}=m_{1}=m_{2}$ and $N_{f}=N_{1}+N_{2}$ flavors. In the context of QCD it is possible to use chiral perturbation theory to calculate the way the masses of mesons and couplings change when small quark masses are modified by small amounts [32,33]. However, so far, this is not the case for $N_{f}=8$ or 12 .

It is commonly believed that for $N_{f}=8$ chiral symmetry is broken spontaneously, however the ratio $M_{\pi}^{2} / m_{f}$ has large nonlinear variations for $0.01<a m_{f}<0.05$ compared to $N_{f}=4$ in the same $a m_{f}$ range, $a$ being the lattice spacing. This can be seen clearly by comparing Figs. 22 and 23 in Ref. [6]. A detailed discussion of the applicability of chiral perturbation theory for $N_{f}=8$ can be found in Sec. IV of Ref. [6], where it is stated that there is no numerical evidence for the predicted chiral logs. For $N_{f}=12$, the situation is more controversial. It is clear that if chiral symmetry is unbroken in the massless limit, the conventional tools are not useful.
Given the lack of reliable ways to calculate the mass dependence of the meson masses and coupling, we followed a phenomenological approach for the unperturbed spectrum [12]. In the limit $m_{1}=m_{2}, v=v_{1}=v_{2}, X v^{N_{f}-2}$, $\lambda_{\sigma} v^{2}$ and $\lambda_{a 0} v^{2}$ can be eliminated in terms of the zerothorder masses. Introducing the notations

$$
\begin{aligned}
\Delta_{\sigma} & \equiv M_{\sigma}^{2}-M_{\pi}^{2} \\
\Delta_{a 0} & \equiv M_{a 0}^{2}-M_{\pi}^{2} \\
\Delta_{\eta^{\prime}} & \equiv M_{\eta^{\prime}}^{2}-M_{\pi}^{2},
\end{aligned}
$$

these relations can be written as

$$
\begin{aligned}
X v^{N_{f}-2} & =\Delta_{\eta^{\prime}} \\
\lambda_{\sigma} v^{2} & =\Delta_{\sigma}+\left(1-2 / N_{f}\right) \Delta_{\eta^{\prime}} \\
\lambda_{a 0} v^{2} & =\Delta_{a 0}-\left(2 / N_{f}\right) \Delta_{\eta^{\prime}} .
\end{aligned}
$$

We also introduced the dimensionless ratios [12]:

$$
\begin{gathered}
R_{\sigma} \equiv \lambda_{\sigma} v^{2} / M_{\eta^{\prime}}^{2}, \\
R_{a_{0}} \equiv \lambda_{a 0} v^{2} / M_{\eta^{\prime}}^{2} .
\end{gathered}
$$




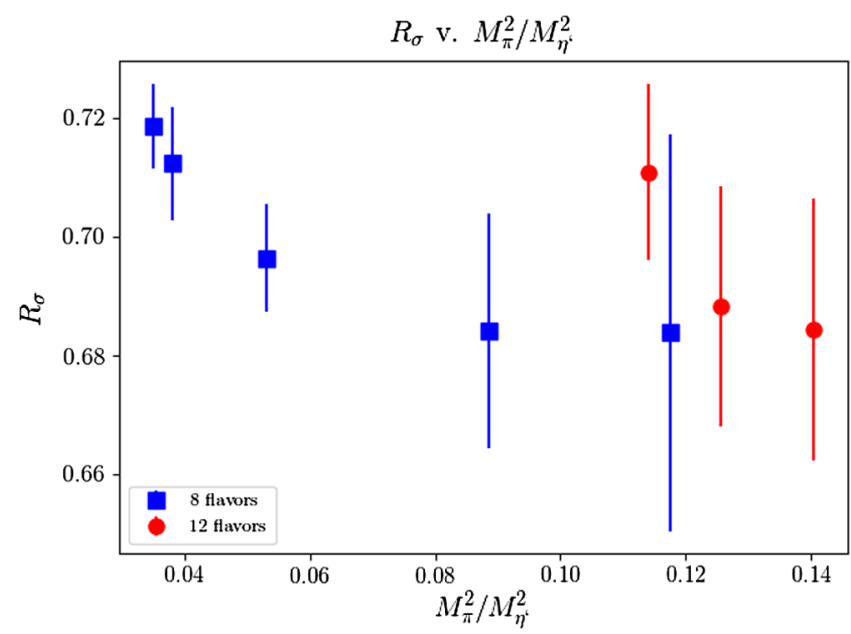

FIG. 1. $R_{\sigma}$ for $N_{f}=8$ (squares) and 12 (circles) versus $\left(M_{\pi} / M_{\eta^{\prime}}\right)^{2}$.

Numerically, dividing by $M_{\eta^{\prime}}^{2}$ has a small effect because $0.87<a M_{\eta^{\prime}}<1.025$ and it removes the explicit dependance on the lattice spacing. This approach fixes the three unknown quantities, $X, \lambda_{a 0}, \lambda_{\sigma}$ with three numerical inputs, $M_{\pi}^{2} / M_{\eta^{\prime}}^{2}, M_{a 0}^{2} / M_{\eta^{\prime}}^{2}$ and $M_{\sigma}^{2} / M_{\eta^{\prime}}^{2}$. Interesting regularities are found for $R_{\sigma}$ and $R_{a_{0}}$. As an order of magnitude we found that for small $m_{f}, R_{\sigma} \simeq 1-2 / N_{f}$ and $R_{a_{0}} \simeq-2 / N_{f}$ with small variations with the fermion mass. Figures 1 and 2 . indicate that mass dependence has regularities that one should try to understand analytically. $R_{\sigma}$ decreases with $\left(M_{\pi} / M_{\eta^{\prime}}\right)^{2}$ while $R_{a_{0}}$ increases. The order of magnitudes of the changes in $R_{\sigma}$ and $R_{a_{0}}$ are roughly the same as those of $\left(M_{\pi} / M_{\eta^{\prime}}\right)^{2}$.

This empirical data shows that in order to fit the spectrum, the quartic couplings used in the tree-level mass formulas need to be tuned as the symmetry breaking term

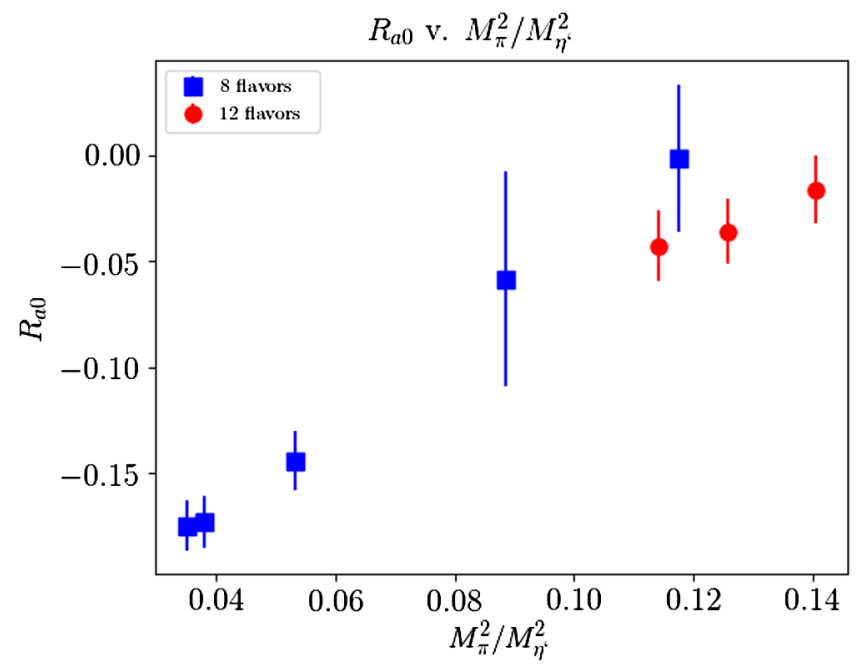

FIG. 2. $R_{a_{0}}$ for $N_{f}=8$ (squares) and 12 (circles) versus $\left(M_{\pi} / M_{\eta^{\prime}}\right)^{2}$. changes. In a similar way, if we change $v_{1}$ and $v_{2}$ significantly, we also need to adjust the quartic coupling. For this reason, we will develop a perturbative approach where these adjustments would appear at second order.

\section{PERTURBATIVE SPLITTINGS}

In this section we consider an infinitesimal perturbation from the limit where $v_{1}=v_{2}=v$ with $v$ the vacuum expectation of $S_{0}$ for the one mass case. In the following, we work at first order in $v_{2}-v_{1}$ and the symbol $\simeq$ denotes equalities valid up to corrections of order $\left(v_{2}-v_{2}\right)^{2}$. Notice that the mixing terms $M_{S_{08}}^{2}$ and $M_{P_{08}}^{2}$ are of first order and have effects of second order on the mass eigenstates of the 2 by 2 matrix of singlets. Consequently mixing will be ignored in this section.

\section{A. Adjoint splittings}

At first order in $v_{2}-v_{1}$, it is possible to express the splittings among the four masses of the scalars and pseudoscalars corresponding to the decomposition of the $S U\left(N_{f}\right)_{V}$ adjoint given in Eq. (20), in terms of the unperturbed masses. For instance,

$$
\begin{aligned}
M_{\pi_{h h}}^{2}-M_{\pi_{l l}}^{2} & =\left(v_{2}^{2}-v_{1}^{2}\right)\left(\frac{\lambda_{a 0}}{2}+\frac{X}{N_{f}} v_{1}^{N_{1}-2} v_{2}^{N_{2}-2}\right) \\
& \simeq \frac{\left(v_{2}-v_{1}\right)}{v}\left(\lambda_{a 0} v^{2}+\frac{2 X}{N_{f}} v^{N_{f}-2}\right) .
\end{aligned}
$$

Using these relations we obtain

$$
M_{\pi_{h h}}^{2}-M_{\pi_{l l}}^{2} \simeq \frac{\left(v_{2}-v_{1}\right)}{v} \Delta_{a 0} .
$$

Proceeding similarly, we obtain at first order:

$$
\begin{gathered}
M_{\pi_{l h}}^{2}-M_{\pi_{l l}}^{2} \simeq \frac{1}{2}\left(M_{\pi_{h h}}^{2}-M_{\pi_{l l}}^{2}\right) \\
M_{P_{88}}^{2}-M_{\pi_{l l}}^{2} \simeq \frac{N_{1}}{N_{f}}\left(M_{\pi_{h h}}^{2}-M_{\pi_{l l}}^{2}\right) .
\end{gathered}
$$

It is possible to treat the scalar splittings in a completely similar way. The results are

$$
\begin{aligned}
& M_{a 0_{h h}}^{2}-M_{a 0_{l l}}^{2} \simeq \frac{\left(v_{2}-v_{1}\right)}{v}\left(3 \Delta_{a 0}-\frac{8}{N_{f}} \Delta_{\eta^{\prime}}\right) \\
& M_{a 0_{l h}}^{2}-M_{a 0_{l l}}^{2} \simeq \frac{1}{2}\left(M_{a 0_{h h}}^{2}-M_{a 0_{l l}}^{2}\right) \\
& M_{S_{88}}^{2}-M_{a 0_{l l}}^{2} \simeq \frac{N_{1}}{N_{f}}\left(M_{a 0_{h h}}^{2}-M_{a 0_{l l}}^{2}\right) .
\end{aligned}
$$

In order to have the expected splittings for $M_{\pi}^{2}$ where the pions containing lighter hyperfermions are lighter, we need 
TABLE I. Values $\frac{\Delta_{a_{0}}}{M_{\eta^{\prime}}^{2}}$ and $\frac{3 \Delta_{a_{0}}-\left(8 / N_{f}\right) \Delta_{\eta^{\prime}}}{M_{\eta^{\prime}}^{2}}$ using the LatKMI data $[6,11]$.

\begin{tabular}{llll}
\hline \hline$N_{f}$ & $\left(a m_{f}\right)$ & \multicolumn{1}{c}{$\frac{\Delta_{a_{0}}}{M_{\eta^{\prime}}}$} & $\frac{3 \Delta_{a_{0}}-\left(8 / N_{f}\right) \Delta_{\eta^{\prime}}}{M_{\eta^{\prime}}^{2}}$ \\
\hline 8 & 0.012 & $0.0584(74)$ & $-0.669(71)$ \\
8 & 0.015 & $0.0644(78)$ & $-0.724(81)$ \\
8 & 0.02 & $0.0885(95)$ & $-0.640(70)$ \\
8 & 0.03 & $0.160(41)$ & $-0.38(16)$ \\
8 & 0.04 & $0.214(28)$ & $-0.22(11)$ \\
12 & 0.04 & $0.0854(94)$ & $-0.225(52)$ \\
12 & 0.05 & $0.1079(65)$ & $-0.163(51)$ \\
12 & 0.06 & $0.1124(73)$ & $-0.261(65)$ \\
\hline \hline
\end{tabular}

to have $v_{2}>v_{1}$ because $\Delta_{a_{0}}>0$ (see Table I). However, this choice implies that the various $\mathbf{a}_{\mathbf{0}}$ appear in the inverse order $\left(M_{a 0_{h h}}^{2}<M_{a 0_{h l}}^{2}<M_{a 0_{l l}}^{2}\right)$ because $\frac{3 \Delta_{a_{0}}-\left(8 / N_{f}\right) \Delta_{\eta^{\prime}}}{M_{\eta^{\prime}}^{2}}<0$ (see Table I) for all the LatKMI datasets $[6,11]$. The negative sign is clearly related to the lightness of the $a_{0}$ compared to QCD.

The reasons for the inversion are clearly visible in Eq. (23). Assuming $v_{2}>v_{1}$ to get the standard ordering for the pseudoscalars, we see that $\lambda_{a_{0}}<0$ makes $\lambda_{a_{0}} v_{2}^{2}$ more negative for $M_{a 0_{h h}}^{2}$. In addition, the anomaly term for $M_{a 0_{h h}}^{2}$ has larger powers of $v_{1}$ and lower powers of $v_{2}$ than $M_{a 0_{l l}}^{2}$ and the coupling is positive so again it inverts the ordering. For the pseudoscalars, the coupling is negative so it is in normal order.

\section{B. Parametrization of $v_{1}$ and $v_{2}$}

So far we have not discussed how $v_{1}$ and $v_{2}$ differ from $v$ and from each other. In general, it is possible to select a path leaving the $v_{1}=v_{2}$ line in the $\left(v_{1}, v_{2}\right)$ plane in such a way that some condition is satisfied. An example of condition that we may like to impose is $\delta M_{\pi_{l l}}^{2}=0$. In the linear approximation this implies a linear relation between the changes.

At first order, we will use the parametrization:

$$
v_{i}=v\left(1+\epsilon g_{i}\right),
$$

for $i=1,2$ and $g_{i}$ to be determined. At first order in $\epsilon$, we have the mass variations with respect to the unperturbed case

$$
\delta M_{\mathrm{mes}}^{2}=\left.\epsilon \sum_{i=1}^{2} g_{i} v \frac{\partial M_{\mathrm{mes}}^{2}}{\partial v_{i}}\right|_{v_{1}=v_{2}=v}
$$

where mes is any of the 10 mesons states. This subscript will be dropped in the following. The expressions
TABLE II. Coefficients $d_{1 a 0}, d_{1 \sigma}$ and $d_{1 \eta^{\prime}}$.

\begin{tabular}{lccc}
\hline \hline & $d_{1 a 0}$ & $d_{1 \sigma}$ & $d_{1 \eta^{\prime}}$ \\
\hline$\delta M_{\pi_{l l}}^{2}$ & $\frac{N_{2}}{N_{f}}$ & $\frac{N_{1}}{N_{f}}$ & 0 \\
$\delta M_{\pi_{l h}}^{2}$ & $\frac{N_{f}-2 N_{1}}{N_{f} 2}$ & $\frac{N_{1}}{N_{f}}$ & 0 \\
$\delta M_{\pi_{h h}}^{2}$ & $-\frac{N_{1}}{N_{f}}$ & $\frac{N_{1}}{N_{f}}$ & 0 \\
$\delta M_{P 88}^{2}$ & $\frac{N_{2}-N_{1}}{N_{f}}$ & $\frac{N_{1}}{N_{f}}$ & 0 \\
$\delta M_{P 00}^{2}$ & 0 & $\frac{N_{1}}{N_{f}}$ & $\frac{N_{1}\left(N_{f}-2\right)}{N_{f}}$ \\
$\delta M_{a 0_{l l}}^{2}$ & $3-\frac{N_{1}}{N_{f}}$ & $\frac{N_{1}}{N_{f}}$ & $\frac{2\left(N_{1}-4\right)}{N_{f}}$ \\
$\delta M_{a 0_{l h}}^{2}$ & $\frac{3}{2}-\frac{N_{1}}{N_{f}}$ & $\frac{N_{1}}{N_{f}}$ & $\frac{2\left(N_{1}-2\right)}{N_{f}}$ \\
$\delta M_{a 0_{h h}}^{2}$ & $-\frac{N_{1}}{N_{f}}$ & $\frac{N_{1}}{N_{f}}$ & $2 \frac{N_{1}}{N_{f}}$ \\
$\delta M_{S 88}^{2}$ & $\frac{4 N_{2}}{N_{f}}-1$ & $\frac{N_{1}}{N_{f}}$ & $\frac{2\left(N_{1} N_{f}-4 N_{2}\right)}{N_{f}^{2}}$ \\
$\delta M_{S 00}^{2}$ & 0 & $\frac{3 N_{1}}{N_{f}}$ & $-\frac{N_{1}\left(N_{f}^{2}-6 N_{f}+8\right)}{N_{f}^{2}}$ \\
\hline \hline
\end{tabular}

$$
\left.d_{i} \equiv v \frac{\partial M^{2}}{\partial v_{i}}\right|_{v_{1}=v_{2}=v},
$$

are evaluated at zeroth-order and are functions of $X v^{N_{f}-2}$, $\lambda_{\sigma} v^{2}$ and $\lambda_{a 0} v^{2}$. This implies that the gradients can be expressed as

$$
d_{i}=d_{i \sigma} \Delta_{\sigma}+d_{i a 0} \Delta_{a 0}+d_{i \eta^{\prime}} \Delta_{\eta^{\prime}} .
$$

The values of these coefficients are listed in Tables II and III for each of the meson states.

For practical applications it may be useful to choose the relation between $g_{1}$ and $g_{2}$ in such a way that some mass stays constant. The case $\delta M_{\sigma}^{2}=0$ is special because $d_{1} / d_{2}=N_{1} / N_{2}$ and consequently we can take $g_{1} / g_{2}=$ $-N_{2} / N_{1}$. This also implies that $\delta M_{\eta^{\prime}}^{2}=0$. This choice is illustrated in Fig. 3. We remind that at first order, mixings

TABLE III. Coefficients $d_{2 a 0}, d_{2 \sigma}$ and $d_{2 \eta^{\prime}}$.

\begin{tabular}{lccc}
\hline \hline & $d_{2 a 0}$ & $d_{2 \sigma}$ & $d_{2 \eta^{\prime}}$ \\
\hline$\delta M_{\pi_{l l}}^{2}$ & $-\frac{N_{2}}{N_{f}}$ & $\frac{N_{2}}{N_{f}}$ & 0 \\
$\delta M_{\pi_{l h}}^{2}$ & $\frac{N_{f}-2 N_{2}}{N_{f} 2}$ & $\frac{N_{2}}{N_{f}}$ & 0 \\
$\delta M_{\pi_{h h}}^{2}$ & $\frac{N_{1}}{N_{f}}$ & $\frac{N_{2}}{N_{f}}$ & 0 \\
$\delta M_{P 88}^{2}$ & $\frac{N_{1}-N_{2}}{N_{f}}$ & $\frac{N_{2}}{N_{f}}$ & 0 \\
$\delta M_{P 00}^{2}$ & 0 & $\frac{N_{2}}{N_{f}}$ & $\frac{N_{2}\left(N_{f}-2\right)}{N_{f}}$ \\
$\delta M_{a 0_{l l}}^{2}$ & $-\frac{N_{2}}{N_{f}}$ & $\frac{N_{2}}{N_{f}}$ & $\frac{2 N_{2}}{N_{f}}$ \\
$\delta M_{a 0_{l h}}^{2}$ & $\frac{3}{2}-\frac{N_{2}}{N_{f}}$ & $\frac{N_{2}}{N_{f}}$ & $\frac{2\left(N_{2}-2\right)}{N_{f}}$ \\
$\delta M_{a 0_{h h}}^{2}$ & $3-\frac{N_{2}}{N_{f}}$ & $\frac{N_{2}}{N_{f}}$ & $\frac{2\left(N_{2}-4\right)}{N_{f}}$ \\
$\delta M_{S 88}^{2}$ & $\frac{3 N_{1}-N_{2}}{N_{f}}$ & $\frac{N_{2}}{N_{f}}$ & $\frac{2\left(N_{2} N_{f}-4 N_{1}\right)}{N_{f}^{2}}$ \\
$\delta M_{S 00}^{2}$ & 0 & $\frac{3 N_{2}}{N_{f}}$ & $-\frac{N_{2}\left(N_{f}^{2}-6 N_{f}+8\right)}{N_{f}^{2}}$ \\
\hline \hline
\end{tabular}




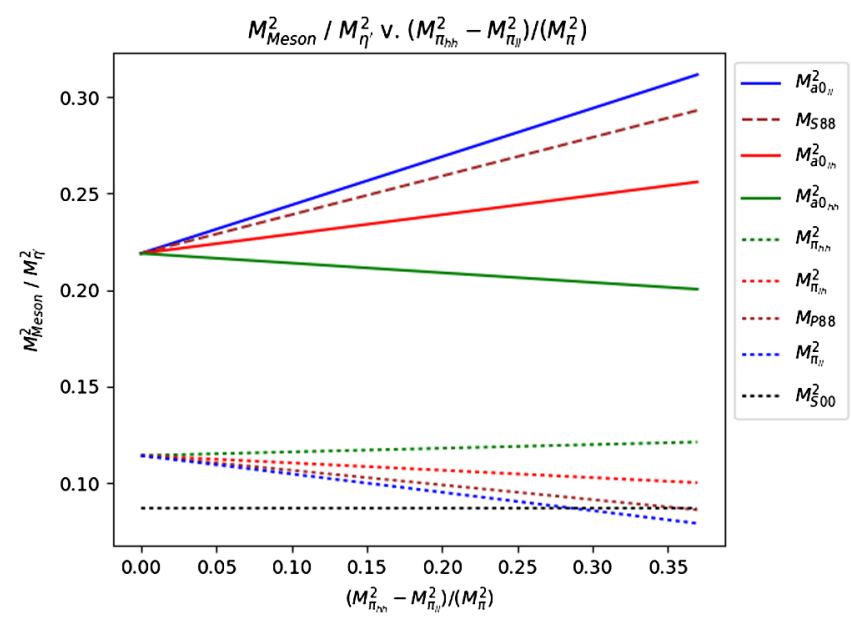

FIG. 3. The spectrum of $M_{\text {meson }}^{2}$ in $M_{\eta^{\prime}}^{2}$ units, in the linear approximation for $N_{1}=2$ and $N_{2}=10$ versus $\left(M_{\pi_{h h}}^{2}-M_{\pi_{l l}}^{2}\right) / M_{\pi}^{2}$. The unperturbed spectrum, which includes the values of $M_{\eta^{\prime}}^{2}$ and $M_{\pi}^{2}$ used in the graph come from the LatKMI data discussed in the text for $N_{f}=12$ and $a m_{f}=0.04$.

are neglected and so $M_{\sigma} \simeq M_{S 00}$ and $M_{\eta^{\prime}} \simeq M_{P 00}$. Another interesting choice is $\delta M_{\pi_{l l}}^{2}=0$. However in this case the mass differences do not cancel in $d_{1} / d_{2}$ and the ratios of $g_{1} / g_{2}$ needs to be adjusted separately for different data sets.

At this point, it is difficult to go beyond first order. Figures 1 and 2 show that in order to fit the tree-level spectra with a varying mass, the values of $\lambda_{a 0}$ and $\lambda_{\sigma}$ need to be tuned accordingly. It is possible that radiative corrections could account for the observed regularities in Figs. 1 and 2 and provide second-order effects. These effects should be combined with the second-order effects in the mass formulas at fixed $\lambda_{a 0}$ and $\lambda_{\sigma}$ provided above. These nonlinear effects can be calculated easily from the explicit formulas. In order to give an idea of the order of magnitude of these effects, we have displayed $M_{a 0_{l l}}^{2}, M_{a 0_{l h}}^{2}$ and $M_{a 0_{h h}}^{2}$ from the complete (nonlinear) formulas Eq. (23) in Fig. 4.

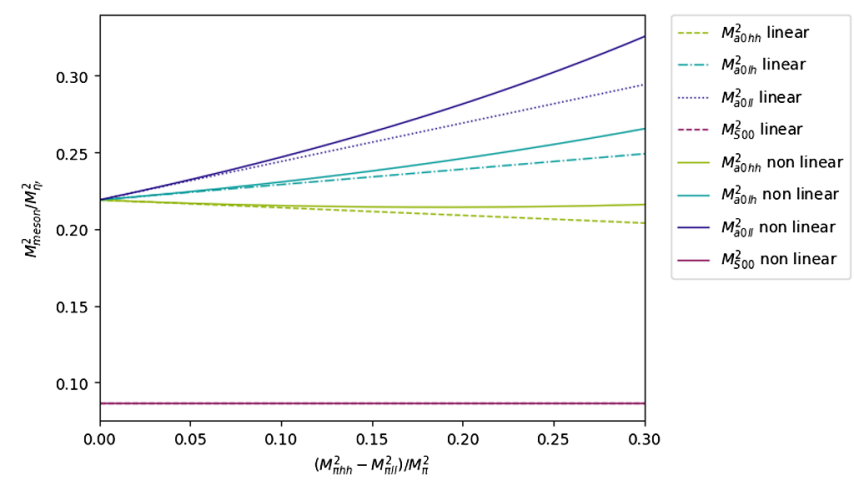

FIG. 4. $\quad M_{a 0_{l l}}^{2}, M_{a 0_{l h}}^{2}$ and $M_{a 0_{h h}}^{2}$ in $M_{\eta^{\prime}}^{2}$ units, using nonlinear formulas versus $\left(M_{\pi_{h h}}^{2}-M_{\pi_{l l}}^{2}\right) / M_{\pi}^{2}$. The unperturbed spectrum, for the same choice of parameters as in Fig. 3. We emphasize that other corrections need to be included to get a realistic graph.
This indicates that $20-40$ percent corrections from higher order effects should be expected in the range of perturbations considered in the figure.

\section{CONCLUSIONS}

In summary, we have calculated the tree level spectrum for a linear model corresponding to a symmetry breaking with two different masses with adjustable multiplicities. We developed a perturbative expansion in the mass difference $\left(M_{\pi_{h h}}^{2}-M_{\pi_{l l}}^{2}\right)$ which provides simple results for the ratios of differences of masses squared $\left(1 / 2\right.$ and $\left.N_{1} / N_{f}\right)$ and have similar structure for the adjoint of the scalar and pseudoscalars. However, when we impose the familiar ordering for the pseudoscalars $\left(M_{\pi_{l l}}^{2}<M_{\pi_{h h}}^{2}\right)$ we obtained the inverse ordering for the scalars $\left(M_{a 0_{l l}}^{2}>M_{a 0_{h h}}^{2}\right)$.

The two possible reasons for the inversion can be seen in Eq. (23) and are $\lambda_{a_{0}}<0$ and the relative sign of the anomaly term in the spectrum. This inversion prediction could be verified or falsified by ongoing multiflavors simulations. The verification would be a surprising and interesting result. Numerical disagreement with the inversion would lead us to reconsider the two underlying reasons for the inversion. It seems clear that if $m_{2} \gg m_{1}$, the normal order should be restored. It is possible that the connection between the two regimes could be understood using the radiative corrections or sudden vacuum changes. In [26], additional degrees of freedom such as glueballs were introduced in the model. This extended model has richer mass formulas which could have implications for the a0 mass inversion.

The question of vacuum stability requires a more detailed study. The negative sign in Eq. (7) makes clear that the potential is not reliable for large values of $v_{i}$. If we set $X=0$, negative values of $\lambda_{a_{0}}$ are compatible with the stability requirement, if $\lambda_{\sigma} \geq\left(N_{f}-1\right)\left|\lambda_{a 0}\right|$ [12]. This bound is satisfied for the three data sets with $N_{f}=12$ and for the two more massive datasets with $N_{f}=8$, but it is violated for the three lightest data sets for $N_{f}=8$. If in addition we set $b_{i}=0$, negative values of $\lambda_{a_{0}}$ produce a different vacuum which is breaking the vector symmetry $S U\left(N_{f}\right)_{V}[34,35]$. However, for the values of $b_{0}$ corresponding to the five asymptotically stable data sets, the proper $S U\left(N_{f}\right)_{V}$-invariant vacuum is restored. These results suggest that for low enough mass and at fixed lattice size, some transition may take place [36].

The inversion could be tested for instance with $N_{1}=2$ and $N_{2}=6$ with $a m_{1}=0.012$ and $a m_{2}=0.015$ which are mass parameters used by LatKMI. The masses are small enough to have a clearly negative $\lambda_{a 0}$ and the relative mass difference seems small enough to avoid large nonlinear corrections. It is interesting to notice that at first order in $\delta m$, the Nussinov inequality [37] $M_{l h} \geq(1 / 2)\left(M_{l l}+M_{h h}\right)$ is saturated despite the inversion. 
In $\mathrm{QCD}$, the $a_{0}$ can decay into $\eta \pi$ and is sometimes considered as a more complicated degree of freedom [20]. However, in the single mass situation with $N_{f}=8$ or 12 , where $\eta$ and $\pi$ are degenerate, the $a_{0}$ of the LatKMI data is light enough to forbid the on-shell process. We expect this property to remain valid with the small mass difference suggested above. This should make the lattice analysis simpler than in QCD.

More generally our work should be considered as an encouragement to calculate spectra with two not so different masses and test model calculations of the effects of the mass difference with a reliability comparable to what can be done with chiral perturbation theory for QCD.

\section{ACKNOWLEDGMENTS}

We thank A. Gasbarro and O. Witzel for discussions. We thank M. Ogilvie for pointing Ref. [37] and D. Hacket for pointing out Ref. [34]. This research was supported in part by the Department of Energy under Award No. DOE grant DE-SC0010113.
[1] B. Svetitsky, in Proceeding of 35th International Symposium on Lattice Field Theory, Granada, Spain (EPD Sciences, 2017), http://inspirehep.net/record/1616208/ files/arXiv:1708.04840.pdf.

[2] T. DeGrand, Rev. Mod. Phys. 88, 015001 (2016).

[3] D. Nogradi and A. Patella, Int. J. Mod. Phys. A 31, 1643003 (2016).

[4] Y. Aoki et al. (LatKMI Collaboration), Phys. Rev. D 89, 111502 (2014).

[5] T. Appelquist, R. C. Brower, G. T. Fleming, A. Hasenfratz, X. Y. Jin, J. Kiskis, E. T. Neil, J. C. Osborn, C. Rebbi, E. Rinaldi, D. Schaich, P. Vranas, E. Weinberg, and O. Witzel (Lattice Strong Dynamics (LSD) Collaboration), Phys. Rev. D 93, 114514 (2016).

[6] Y. Aoki et al. (LatKMI Collaboration), Phys. Rev. D 96, 014508 (2017).

[7] A. D. Gasbarro and G. T. Fleming, Proc. Sci., LATTICE2016 (2017) 242 [arXiv:1702.00480].

[8] Y. Aoki, T. Aoyama, M. Kurachi, T. Maskawa, K.-i. Nagai, H. Ohki, E. Rinaldi, A. Shibata, K. Yamawaki, and T. Yamazaki (LatKMI Collaboration), Phys. Rev. Lett. 111, 162001 (2013).

[9] Z. Fodor, K. Holland, J. Kuti, S. Mondal, D. Nogradi, and C. H. Wong, Proc. Sci., LATTICE2014 (2015) 244 [arXiv:1502.00028].

[10] Y. Aoki, Flavor singlet mesons in qcd with varying number of flavors, Proceedings of Lattice 2016 on behalf of the LatKMI Collaboration, (EPD Sciences, 2016), https:// conference.ippp.dur.ac.uk/event/470/contributions/2399/ attachments/2186/2414/slide_yaoki_Lattice2016.pdf.

[11] Y. Aoki et al., EPJ Web Conf. 175, 08023 (2018).

[12] Y. Meurice, Phys. Rev. D 96, 114507 (2017).

[13] T. Banks and A. Zaks, Nucl. Phys. B196, 189 (1982).

[14] F. Sannino and K. Tuominen, Phys. Rev. D 71, 051901 (2005).

[15] D. D. Dietrich and F. Sannino, Phys. Rev. D 75, 085018 (2007).
[16] J. Schechter and Y. Ueda, Phys. Rev. D 3, 2874 (1971).

[17] C. Rosenzweig, J. Schechter, and C. G. Trahern, Phys. Rev. D 21, 3388 (1980).

[18] G. 't Hooft, Phys. Rep. 142, 357 (1986).

[19] Y. Meurice, Mod. Phys. Lett. A 02, 699 (1987).

[20] A. H. Fariborz, R. Jora,, and J. Schechter, Phys. Rev. D 77, 094004 (2008).

[21] D. Parganlija, F. Giacosa, and D. H. Rischke, Phys. Rev. D 82, 054024 (2010).

[22] D. Parganlija, P. Kovács, G. Wolf, F. Giacosa, and D. H. Rischke, Phys. Rev. D 87, 014011 (2013).

[23] P. Kovács and G. Wolf, Acta Phys. Pol. B Proc. Suppl. 6, 853 (2013).

[24] P. Kovács and Z. Szép, Phys. Rev. D 75, 025015 (2007).

[25] P. Kovács, Z. Szép, and G. Wolf, Phys. Rev. D 93, 114014 (2016).

[26] A. H. Fariborz and R. Jora, arXiv:1807.10927 [Phys. Rev. D (to be published)].

[27] C. Csaki, C. Grojean,, and J. Terning, Rev. Mod. Phys. 88, 045001 (2016).

[28] A. Hasenfratz, C. Rebbi, and O. Witzel, Phys. Lett. B 773, 86 (2017).

[29] R. C. Brower, A. Hasenfratz, C. Rebbi, E. Weinberg, and O. Witzel, Phys. Rev. D 93, 075028 (2016).

[30] T. Ma and G. Cacciapaglia, J. High Energy Phys. 03 (2016) 211.

[31] S. Weinberg, The Quantum Theory of Fields, Modern Applications Vol. 2 (Cambridge University Press, Cambridge, England, 2013).

[32] S. Scherer and M. R. Schindler, arXiv:hep-ph/0505265.

[33] J. Bijnens, Prog. Part. Nucl. Phys. 58, 521 (2007).

[34] A. Butti, A. Pelissetto, and E. Vicari, J. High Energy Phys. 08 (2003) 029.

[35] D. C. Hackett, Phys. Rev. D 97, 055050 (2018).

[36] T. Appelquist et al. (Lattice Strong Dynamics Collaboration), arXiv:1807.08411.

[37] S. Nussinov, Phys. Rev. Lett. 52, 966 (1984). 